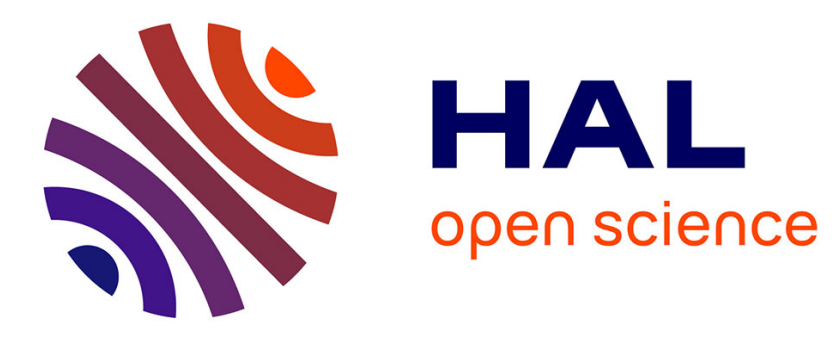

\title{
New concepts in the management of chronic cough
}

Surinder S. Birring

\section{To cite this version:}

Surinder S. Birring. New concepts in the management of chronic cough. Pulmonary Pharmacology \&

Therapeutics, 2011, 24 (3), pp.334. 10.1016/j.pupt.2011.01.005 . hal-00750479

\section{HAL Id: hal-00750479 \\ https://hal.science/hal-00750479}

Submitted on 10 Nov 2012

HAL is a multi-disciplinary open access archive for the deposit and dissemination of scientific research documents, whether they are published or not. The documents may come from teaching and research institutions in France or abroad, or from public or private research centers.
L'archive ouverte pluridisciplinaire HAL, est destinée au dépôt et à la diffusion de documents scientifiques de niveau recherche, publiés ou non, émanant des établissements d'enseignement et de recherche français ou étrangers, des laboratoires publics ou privés. 


\section{Accepted Manuscript}

Title: New concepts in the management of chronic cough

Authors: Surinder S. Birring, MD

PII: S1094-5539(11)00009-5

DOI: $\quad$ 10.1016/j.pupt.2011.01.005

Reference: $\quad$ YPUPT 1074

To appear in: Pulmonary Pharmacology \& Therapeutics

Received Date: 30 November 2010

Revised Date: 31 December 2010

Accepted Date: 12 January 2011

Please cite this article as: Birring SS. New concepts in the management of chronic cough, Pulmonary Pharmacology \& Therapeutics (2011), doi: 10.1016/j.pupt.2011.01.005

This is a PDF file of an unedited manuscript that has been accepted for publication. As a service to our customers we are providing this early version of the manuscript. The manuscript will undergo copyediting, typesetting, and review of the resulting proof before it is published in its final form. Please note that during the production process errors may be discovered which could affect the content, and all legal disclaimers that apply to the journal pertain. 
New concepts in the management of chronic cough

Surinder S Birring MD

King's College London, Division of Asthma, Allergy and Lung Biology, London, United Kingdom.

Correspondence and request for reprints to:

Dr Surinder S Birring

King's College London,

Division of Asthma, Allergy and Lung Biology,

Denmark Hill,

London.

SE5 9RS.

E-mail: surinder.birring@nhs.net

Telephone: (+ 44) 2032999000

Fax: $\quad$ (+44) 2032993791 


\begin{abstract}
There has been significant progress in the field of cough in the past 10 years. Obstructive sleep apnoea, tonsillar enlargement and environmental fungi have recently been described as causes of chronic cough. The advances in the assessment of gastro-oesophageal reflux (GOR) have led to a greater understanding of the relationship between cough and GOR and are likely to change the approach to managing patients with GOR-cough. The investigation of the phenotype of patients with idiopathic chronic cough has provided novel insights. Patients with an idiopathic chronic are predominantly female, have an onset of cough around the menopause and have a high prevalence of organ specific autoimmune disease, particularly hypothyroidism. The presence of bronchoalveolar lymphocytosis suggests there is homing of inflammatory cells from primary sites of autoimmune inflammation to the lungs. The importance of heightened cough reflex sensitivity has led to some investigators to suggest that chronic cough be recognised as unique entity called Cough Hypersensitivity Syndrome (CHS). A number of subjective and objective tools have been developed recently to assess cough severity. Antitussive drug development and their assessment in randomised controlled clinical trials is an emerging and exciting area of cough research.
\end{abstract}




\section{Introduction}

The management of patients with chronic cough is challenging on many fronts. The evaluation of patients involves numerous investigations and treatment trials. The optimal approach is not known, for example, the evidence for the role of proton pump inhibitor therapy for gastrooesophageal reflux associated chronic cough is conflicting. ${ }^{1}$ A significant number of patients with chronic cough remain unexplained. The reasons for this are unclear and there are few effective treatment options. Cough reflex hypersensitivity is a key feature of chronic cough but the mechanism of sensitisation is not known and it currently has little role in the management of patients. Few randomised controlled trials have been performed in chronic cough to guide clinical practice. The evaluation of antitussive drugs until recently had been hampered by a lack of validated and objective tools to assess cough severity and the difficulty in interpreting data in a clinically meaningful way. This review will discuss some of the recent advances in the field of cough that begin to address some of the key challenges.

\section{New chronic cough conditions}

The recent identification of a number of conditions associated with unexplained chronic cough may lead to new therapeutic options for appropriately selected patients. (table 1).

\section{Obstructive sleep apnoea}


A recent study has reported that cough can be the sole manifestation of obstructive sleep apnoea $(\mathrm{OSA}){ }^{2}$ Patients with OSA-cough were likely to be female, overweight, snorers and have symptoms of GOR. ${ }^{3}$ The prevalence of OSA-cough is high when obesity is common in the general population. In a study investigating patients referred for investigation of cough in Utah, USA, a population with a high prevalence of obesity, $44 \%$ of patients had evidence of OSA. ${ }^{4}$ OSA was rarely suspected at presentation and could not be identified from the characteristics of cough alone. Initial therapy for cough was usually directed towards common causes of cough such as asthma, GOR and rhinitis and was not effective. OSA was diagnosed with polysomnography. A trial of continuous positive airway pressure therapy in a small number of patients reported a significant reduction or resolution in cough. ${ }^{2}$ The mechanism of cough in patients with OSA is unclear. There are at least two possibilities. The increase in transdiaphragmatic pressure during episodes of apnoea is thought to cause lower oesophageal sphincter insufficiency leading to GOR. Another potential mechanism is that cough results from upper airway inflammation associated with epithelial injury associated with snoring and apnoeas. The optimal therapy for this group of patients is not known and needs to be investigated in a controlled trial.

\section{Chronic tonsillar enlargement}

The examination of the upper airways is a routine part of the assessment of patients with chronic cough. A recent study reported that a small number of patients with otherwise unexplained chronic cough had enlarged tonsils found coincidentally during examination. ${ }^{5}$ All patients underwent tonsillectomy which led to a reduction or resolution in cough and cough reflex 
hypersensitivity. The histological examination of tonsillar tissue demonstrated chronic inflammation. The mechanism of cough was thought to be related to upper airway inflammation associated with tonsillar enlargement. A similar association between cough and enlarged tonsils has been reported in children. ${ }^{6}$ Further studies need to investigate the efficacy of tonsillectomy in a larger number of patients.

\section{$\underline{\text { Basidiomycetous fungi }}$}

A recent report from Japan has found evidence of environmental basidiomycetous fungi such as Bjerkandera adusta in the airways of patients with unexplained chronic cough. ${ }^{7}$ Basidiomycetous fungi were cultured in induced sputum of approximately $25 \%$ of patients with chronic cough, occurring largely in patients with unexplained chronic cough compared with explained cough. An improvement in cough symptoms with antifungal drug itraconazole has been reported in a controlled trial, suggesting that basidiomycetous fungi may cause cough rather than just colonise the airways. ${ }^{8}$ The association between cough and fungal infection has been termed fungal associated chronic cough (FACC). Further studies are needed to investigate the importance of environmental fungi in cough, particularly outside Japan.

\section{Advances in gastro-oesophageal reflux-cough}

GOR-cough is one of the most commonly reported causes of chronic cough. The cornerstone of therapy for GOR-cough is proton pump inhibitors (PPI). A recent Cochrane meta-analysis review has highlighted that whilst observational studies report a reduction in cough severity with 
PPIs, a number of randomised controlled trials have not confirmed this. ${ }^{1}$ A limitation of the studies included in the Cochrane meta-analysis was that they contained small numbers of patients. Fathi and colleagues have recently reported a larger randomised controlled trial of esmoprazole in 56 patients with chronic cough and GOR and found that esmoprazole was not superior to placebo. ${ }^{9}$ The role of PPIs in chronic cough is questionable and a large multicentre study is needed to provide definitive guidance.

The lack of benefit of PPIs for GOR-cough in some studies raises the possibility that non-acid (or weak acid) GOR may be more important than acid GOR. It has not been possible to study non-acid GOR in cough until recent advances in oesophageal impendence probe technology. Oesophageal impedance probes can differentiate solid, liquid and gaseous oesophageal contents from their electrical impedance characteristics. They can therefore detect non-acid GOR in addition to acid-GOR. The simultaneous development of cough monitoring devices has also contributed to the understanding of the relationship between GOR and cough. Oesophageal impedance studies of patients with chronic cough have found that the number of episodes of both acid and non-acid GOR are not raised compared to healthy subjects. ${ }^{10}$ Studies investigating the temporal relationship between episodes of non-acid GOR and cough have reported that GOR is causally related to cough in only a small number of patients. ${ }^{11}$ Furthermore, in patients with a positive GOR-cough temporal association, Smith et al found that a significant number of cough episodes were unrelated to GOR and therefore it is unlikely that amelioration of GOR will lead to resolution of cough. ${ }^{12}$ It is likely that combined oesophageal impedance-cough monitoring recordings will have a role in the investigation of patients with chronic cough in future. Further 
studies are needed to determine the sensitivity and specificity of oesophageal impedance monitoring for establishing the diagnosis and predicting the response to therapy.

\section{Insights into the mechanisms of idiopathic chronic cough}

\section{Clinical characteristics}

Up to $42 \%$ of patients with chronic cough remain unexplained in specialist clinics and they represent a significant challenge. ${ }^{13}$ The striking phenotype of patients with idiopathic chronic cough (ICC) suggests a unique underlying disorder. The defining features of ICC are patients with chronic cough duration greater than 8 weeks and negative investigations and therapeutic trials for asthma, GOR and rhinitis. Clinical examination, chest radiograph and spirometry by definition are normal. Patients are predominantly female, up to $80 \%$ in some reported series. ${ }^{14}$ The onset of cough tends to be around the menopause. ${ }^{15}$ Many patients report an acute upper respiratory tract infection preceding the onset of chronic cough. ${ }^{13}$ Some patients report an irritation or "itch" in their throat preceding episodes of cough. Common triggers for cough include cold air, strong odors, eating, speech and exercise. Most patients find the cough distressing and have a significantly impaired health related quality of life. ${ }^{16}$ Symptoms of chest pain, stress related urine incontinence, sore throat, social embarrassment and adverse impact on family and partners are common.

Idiopathic chronic cough and autoimmune disease 
A case report of a patient with ICC and autoimmune disease and subsequent studies have led to the hypothesis that ICC may result from autoimmune airway inflammation in a subgroup of patients. ${ }^{17}$ A patient presenting with ICC was incidentally diagnosed with coeliac disease during investigations for cough. All respiratory investigations were normal other than a striking bronchoalvoelar lavage lymphocytosis. The cough and airway lymphocytosis resolved after a trial of gluten free diet. This observation led to the hypothesis that the cough may be associated with other organ specific autoimmune diseases and the mechanism may be homing of lymphocytes from primary sites of autoimmune inflammation (in this case bowel) to the lung. A subsequent case-control study confirmed that the prevalence of autoimmune disease was 8 times higher in ICC compared with matched controls. ${ }^{18}$ The most striking association was with hypothyroidism. A number of studies have since reported a bronchoalveolar lymphocytosis in patients with ICC. ${ }^{14 ; 15}$ Not all patients with ICC had a history of autoimmune disease which raises the possibility that cough may be the first manifestation of autoimmune disease or due to an "autoimmune bronchitis." The absence of significant lymphocytosis in most patients with an explained chronic cough suggests that airway lymphocytosis is unlikely to be caused by the mechanical effects of cough. The association between cough and autoimmune disease is also supported by a 2 to 3 fold increase in cough symptoms in patients with treated hypothyroidism compared with controls. ${ }^{19}$ Furthermore, studies investigating airway function of patients with hypothyroidism have found evidence of airway inflammation and heightened cough reflex sensitivity. ${ }^{20} \mathrm{~A}$ high prevalence of autoimmune disease has also been reported in patients with unexplained fixed airflow obstruction. ${ }^{21}$ These patients are also predominantly female but are significantly older than patients with ICC. An intriguing hypothesis is that airflow obstruction 
may be a long term sequelae of ICC. Long term follow up studies of patients with ICC are needed to address this hypothesis.

A change in airway $\mathrm{T}$ lymphocyte phenotype may be important in the pathogenesis of ICC in addition to increased numbers. An increase in CD4 to CD8 ratio has been reported in healthy women after they go through the menopause which may be important in the development of female predominant respiratory diseases such as cough. ${ }^{22}$ The impact of hormone replacement therapy in cough is not known and is worth investigating. Corticosteroid therapy is ineffective in patients with ICC and organ specific autoimmune disease since most will have had a trial of inhaled or oral corticosteroids. The example of ICC in the patient with coeliac disease suggests that therapy directed at reducing autoimmune lymphocytic inflammation in the primary site can effectively treat cough; this concept deserves further investigation.

\section{Cough hypersensitivity syndrome (CHS): the new paradigm for chronic cough}

Cough reflex hypersensitivity is a key feature in most patients with chronic cough. ${ }^{23}$ It can be assessed by a variety of tussive agents used in cough challenge tests agents such as capsaicin, citric acid, tartaric acid and fog. A wide range of environmental stimuli can trigger cough by activating cough receptors that sense heat, acid, stretch and noxious stimuli. There is evidence of increased cough receptor expression in the airways of patients with cough. Transient receptor potential vanilloid-1 ion channel (TRPV-1) is one example; it is expressed on airway c-nerve fibres and is important in capsaicin induced reflex cough. ${ }^{24}$ Cough reflex hypersensitivity can be 
reversible when cough is caused by infection, eosinophilic airway diseases such as asthma and eosinophilic bronchitis and angiotensin converting enzyme inhibitor drug use. In most patients with chronic cough however, cough reflex hypersensitivity is persistent. Open label studies of therapies that down regulate neural activity such as amitriptyline and gabapentin show promise and have reported a reduction in cough severity. ${ }^{25}$ This needs to be investigated in a randomised controlled trial.

The current diagnostic paradigm focuses on investigating the 3 most common causes of chronic cough: GOR, rhinitis and asthma. The prevalence of unexplained cough in many cough clinics is high despite heightened awareness of the optimal management of cough since the publication of several international guidelines. This has led some investigators to propose a new approach to cough is worth exploring. ${ }^{26}$ It is likely that a number of mechanisms may lead to cough such as cough reflex hypersensitivity, airway hyperresponsiveness, hypersecretion and inflammation. Some patients may have multiple mechanisms for cough. Patients with asthma or chronic obstructive pulmonary disease who cough provide support for this concept; only a subgroup have a hypersensitive cough reflex. ${ }^{23}$ This raises the possibility that the identification of cough reflex hypersensitivity may be useful. A new paradigm in which cough hypersensitivity is the key abnormality in chronic cough has been proposed (Figure 1). ${ }^{27,28}$ Cough hypersensitivity syndrome has been proposed as a term for patients with chronic cough associated with cough reflex hypersensitivity that cannot be explained by other causes (Table 2). This includes most patients with idiopathic chronic cough. It is likely that there will be many subgroups or phenotypes within CHS. The recognition of cough as a unique condition is likely to have a 
positive impact on the evaluation of patients with chronic cough, in contrast to the current perception of cough as a symptom of other disorders such as GOR and rhinitis. Another benefit of this strategy is that it may lead to greater investment into researching why airway nerves are abnormal in cough and the identification of new targets for antitussive drug therapies. It is the author's view that the evaluation of patients with unexplained airway symptoms should include an assessment of airway responsiveness, inflammation and cough reflex hypersensitivity in order to target therapy to the underlying mechanisms. This contrasts to the current clinical practice that largely involves investigation with non-specific tests and trials of therapy. A potential limitation of the evaluation of cough based on cough hypersensitivity is that it doesn't determine the aetiology of cough. The investigation of the factors that sensitise the cough reflex remains a priority.

\section{Advances in cough outcome measures}

The assessment of cough has seen the most rapid and exciting developments in the last 5 years. The assessment of cough severity with validated tools is essential to the development of new therapies. Well established tools such as cough diary scores and visual analogues scales can now be complemented with health related quality of life (QOL) measures and objective cough monitoring. Although cough reflex testing has been used to determine the efficacy of drugs in the past due to the lack of objective tools, in future, it is more likely to be limited to the investigation of the mechanism of action of drugs. 
QOL patient reported outcome measures

Three well validated cough specific QOL questionnaires are available that can assess the patients perspective of living with cough. The Leicester Cough Questionnaire (LCQ), Cough Specific QOL Questionnaire (CQLQ) and Chronic Cough Impact Questionnaire (CCIQ) consists of 19, 28 and 25 items respectively. ${ }^{16 ; 29 ; 30}$ The brevity of the questionnaires means that some important symptoms associated with cough are not featured. All 3 questionnaires are deliberately brief to be practical for clinical and research use. They are designed and validated to quantify QOL numerically with the least number of items and are not intended to identify all adverse impacts associated with cough as obtained with a detailed clinical history. The impact of adverse symptoms not featured in QOL questionnaires are captured by their influence on other important aspects of life and social well-being such as mood and embarrassment. Female patients report worse QOL compared with males when assessed with the LCQ or CQLQ and the reasons for this are unclear. ${ }^{31}$ The minimal important differences (MID) for the LCQ and CQLQ are 1.3 and 22 (maximum possible changes 18 and 84 respectively). ${ }^{32 ; 33}$ The MID for the CQLQ is proportionately larger than that for the LCQ. The MID for the LCQ was determined by methodology commonly used by QOL experts, in which patients were asked to rate the change in their health related QOL using global change rating scales. ${ }^{34}$ The CQLQ MID was determined by asking patients to rate their QOL at two separate visits on visual analogue scales known as the Punum ladder rather than asking patients to rate the change in QOL. The differences in the MID between the 2 instruments are likely to result from the methodology used. The definition of the MID using the Punum ladder encompasses a larger change in QOL than that of the global change rating scales. The initial experience of using the LCQ and CQLQ in randomised controlled trials has been positive. Both questionnaires were practical, had good completion rates and the data 
was clinically meaningful. ${ }^{35}$ They are likely to be standard end points in clinical trials and may help to obtain regulatory approval for new antitussive drugs.

\section{Cough frequency monitors}

The recent advances in sound recording devices and improved battery life have led to the development of a number of cough monitoring devices..$^{12 ; 36-38}$ Many monitors are not validated or commercially available and represent work in progress. It is difficult to review and compare the characteristics of cough monitors because of the lack information or publications relating to validation and accuracy. The Leicester Cough Monitor (LCM) is an automated cough monitor that comprises of a MP3 recorder and a microphone. ${ }^{36: 39}$ It measures cough frequency in the patients' own environment. It has a sensitivity and specificity of $91 \%$ and $99 \%$ respectively for detecting cough in patients with chronic cough. The LCM was independently tested against 24hour cough recordings analysed manually and compared favourably (correlation coefficient $\mathrm{r}=$

0.97). ${ }^{40}$ Coughcount is another automated, sound based cough monitoring system. ${ }^{38}$ Coughcount has not been validated in patients with chronic cough in their own environment; further studies are needed to address this. The VitaloJak is a sound based cough monitor that requires manual analysis of cough recordings. ${ }^{12}$ This is likely to be a labour intensive process even with customized editing software. The cough analysis with this method is likely to be accurate because coughs are manually verified. The LCM and VitaloJak have been the most evaluated cough monitoring systems in patients with chronic cough and report a very similar range of cough frequency data. 
Cough frequency monitors have a number of characteristics that suggest they are likely to be valuable in the clinic and research. Cough frequency is a reported to be repeatable and responsive outcome measure and therefore could be used to assess the efficacy of therapeutic trials. ${ }^{41}$ Cough frequency in healthy subjects is very low compared with patients with chronic cough. Cough monitors can therefore be used to validate the presence of abnormal cough. Recent clinical trials in chronic cough using cough monitoring outcome measures have demonstrated the feasibility of using this technology to assess drug therapy. ${ }^{42}$ It is likely that positive cough monitoring data will provide strong support for regulatory approval of antitussive drugs in future.

\section{Antitussive therapy}

A number of randomised controlled trials have recently been performed in patients with chronic cough; they represent a new era of cough research. The advances in the development of cough outcome measures have facilitated these studies. Morphine, codeine, erythromycin, esomeprazole, itraconazole, zafirlukast and nociceptin opioid like receptor agonists (NOP1) are amongst some of the drugs that have been evaluated in patients with chronic cough. ${ }^{8 ; 9 ; 35 ; 42-44}$ Many lessons have been learned from these studies. Both QOL and objective cough monitoring tools are practical tools and necessary to capture the overall impact of a therapy on cough. The placebo response is significant and therefore needs to be considered in the study design. The ideal group of patients to evaluate an antitussive drug are those with an unexplained chronic cough rather those with a chronic cough due to other respiratory diseases such as asthma or chronic obstructive pulmonary disease. The mechanism of cough in the latter group is likely to differ significantly and may therefore respond differently to therapy. The recruitment of patients 
with unexplained chronic cough into trials has not presented any significant problems and this condition represents a large market and unmet clinical need.

The development of pharmacological therapies for cough can be broadly divided into 2 categories: direct and indirect antitussives. Morphine is thought to act directly on the cough reflex. It has been shown to reduce cough severity in patients with idiopathic chronic cough. ${ }^{35}$ The mechanism of action is thought to be central since it did not reduce peripheral cough reflex hypersensitivity. The use of morphine is limited due to its side effects and risk of dependence. Codeine is another direct antitussive drug. It has not been evaluated in a randomised controlled trial in patients with chronic cough. In patients with subacute post viral cough and COPD, it is ineffective. ${ }^{44: 45}$ The NOP1 agonist SCH486757 has antitussive properties in animal models and is thought to have fewer side effects compared to conventional opiates. In a randomised controlled trial in patients with a post viral subacute cough, the NOP1 agonist was not superior to placebo or codeine. ${ }^{45}$ Indirect antitussive drugs include those that suppress the cough reflex indirectly such as drugs with anti-inflammatory activity. The leukotriene antagonist, Zafirlukast, has been shown to reduce cough hypersensitivity and is recommended for treatment of cough variant asthma. ${ }^{43}$ Erythromycin, an inhibitor of neutrophilic inflammation was ineffective at reducing cough severity in patients with idiopathic chronic cough.[ref] Airway inflammation is an important mechanism of cough in animal models but in humans, its importance is unclear with the exception of corticosteroid therapy for eosinophilic bronchitis. The development of antitussive drugs in future should perhaps focus more on direct pathways, particularly the airway nerves. 
There are non-pharmacological therapeutic options for patients with cough. A speech and language program designed to suppress cough and reduce vocal cord dysfunction and voice abnormalities was highly effective.[ref] This should be recommended for patients with unexplained chronic cough until effective pharmacological therapies are available.

\section{Conclusions}

We now have the tools to do high quality research studies and clinical trials to address areas of uncertainty in cough. Clinicians and researchers have an exciting opportunity to explore new approaches to cough and develop much needed antitussive drug therapy. This will require raising the profile of cough amongst research funders, the pharmaceutical industry and patients. 


\section{References}

1. Chang, A. B., T. J. Lasserson, T. O. Kiljander, F. L. Connor, J. T. Gaffney, and L. A. Garske. 2006. Systematic review and meta-analysis of randomised controlled trials of gastro-oesophageal reflux interventions for chronic cough associated with gastrooesophageal reflux. BMJ 332:11-17.

2. Birring, S. S., A. J. Ing, K. Chan, G. Cossa, S. Matos, M. D. Morgan, and I. D. Pavord. 2007. Obstructive sleep apnoea: a cause of chronic cough. Cough. 3:7.

3. Chan, K. K., A. J. Ing, L. Laks, G. Cossa, P. Rogers, and S. S. Birring. 2010. Chronic cough in patients with sleep-disordered breathing. Eur.Respir J 35:368-372.

4. Sundar, K. M., S. E. Daly, M. J. Pearce, and W. T. Alward. 2010. Chronic cough and obstructive sleep apnea in a community-based pulmonary practice. Cough. 6:2.

5. Birring, S. S., C. Passant, R. B. Patel, B. Prudon, G. E. Murty, and I. D. Pavord. 2004. Chronic tonsillar enlargement and cough: preliminary evidence of a novel and treatable cause of chronic cough. Eur.Respir J 23:199-201.

6. Lewis, M., J. E. McClay, and P. Schochet. 2000. Lingual tonsillectomy for refractory paroxysmal cough. Int.J.Pediatr.Otorhinolaryngol. 53:63-66.

7. Ogawa, H., M. Fujimura, Y. Takeuchi, and K. Makimura. 2009. The importance of basidiomycetous fungi cultured from the sputum of chronic idiopathic cough: a study to 
determine the existence of recognizable clinical patterns to distinguish CIC from nonCIC. Respir Med. 103:1492-1497.

8. Ogawa, H., M. Fujimura, Y. Takeuchi, and K. Makimura. 2009. Efficacy of itraconazole in the treatment of patients with chronic cough whose sputa yield basidiomycetous fungifungus-associated chronic cough (FACC). J Asthma 46:407-412.

9. Fathi, H., R. Faruqi, R. Thompson, C. Wright, and A. H. Morice. 2008. Chronic cough and esomeprazole: a double-blind, randomised placebo-controlled parellel study. Thorax 63:S47.

10. Decalmer, S., R. Stovold, H. jones, J. Pearson, C. Ward, L. A. Houghton, A. Kelsall, K. McGuinness, A. A. Woodcock, and J. A. Smith. 2008. Relationships between microaspiration, gastro-oesophageal reflux and cough in chronic cough subjects. Thorax 63:S46.

11. Blondeau, K., L. J. Dupont, V. Mertens, J. Tack, and D. Sifrim. 2007. Improved diagnosis of gastro-oesophageal reflux in patients with unexplained chronic cough. Aliment.Pharmacol.Ther. 25:723-732.

12. Smith, J. A., S. Decalmer, A. Kelsall, K. McGuinness, H. Jones, S. Galloway, A. Woodcock, and L. A. Houghton. 2010. Acoustic cough-reflux associations in chronic cough: potential triggers and mechanisms. Gastroenterology 139:754-762.

13. Haque, R. A., O. S. Usmani, and P. J. Barnes. 2005. Chronic idiopathic cough: a discrete clinical entity? Chest 127:1710-1713. 
14. Birring, S. S., C. E. Brightling, F. A. Symon, S. G. Barlow, A. J. Wardlaw, and I. D. Pavord. 2003. Idiopathic chronic cough: association with organ specific autoimmune disease and bronchoalveolar lymphocytosis. Thorax 58:1066-1070.

15. Mund, E., B. Christensson, R. Gronneberg, and K. Larsson. 2005. Noneosinophilic CD4 lymphocytic airway inflammation in menopausal women with chronic dry cough. Chest 127:1714-1721.

16. Birring, S. S., B. Prudon, A. J. Carr, S. J. Singh, M. D. Morgan, and I. D. Pavord. 2003. Development of a symptom specific health status measure for patients with chronic cough: Leicester Cough Questionnaire (LCQ). Thorax 58:339-343.

17. Brightling, C. E., F. A. Symon, S. S. Birring, A. J. Wardlaw, R. Robinson, and I. D. Pavord. 2002. A case of cough, lymphocytic bronchoalveolitis and coeliac disease with improvement following a gluten free diet. Thorax 57:91-92.

18. Birring, S. S., A. C. Murphy, J. E. Scullion, C. E. Brightling, M. Browning, and I. D. Pavord. 2004. Idiopathic chronic cough and organ-specific autoimmune diseases: a casecontrol study. Respir Med 98:242-246.

19. Birring S.S., Morgan A.J., Prudon B, McKeever T.M., Lewis S.A., Falconer Smith J.F., Robinson R.J., Britton J.R., and Pavord ID. 2003. Respiratory symptoms in patients with treated hypothyroidism and inflammatory bowel disease. Thorax 58:533-536. 
20. Birring, S. S., R. B. Patel, D. Parker, S. McKenna, B. Hargadon, W. R. Monteiro, J. F. Falconer Smith, and I. D. Pavord. 2005. Airway function and markers of airway inflammation in patients with treated hypothyroidism. Thorax 60:249-253.

21. Birring, S. S., C. E. Brightling, P. Bradding, J. J. Entwisle, D. D. Vara, J. Grigg, A. J. Wardlaw, and I. D. Pavord. 2002. Clinical, radiologic, and induced sputum features of chronic obstructive pulmonary disease in nonsmokers: a descriptive study. Am.J.Respir.Crit Care Med. 166:1078-1083.

22. Mund, E., B. Christensson, K. Larsson, and R. Gronneberg. 2001. Sex dependent differences in physiological ageing in the immune system of lower airways in healthy non-smoking volunteers: study of lymphocyte subsets in bronchoalveolar lavage fluid and blood. Thorax 56:450-455.

23. Prudon, B., S. S. Birring, D. D. Vara, A. P. Hall, J. P. Thompson, and I. D. Pavord. 2005. Cough and glottic-stop reflex sensitivity in health and disease. Chest 127:550-557.

24. Groneberg, D. A., A. Niimi, Q. T. Dinh, B. Cosio, M. Hew, A. Fischer, and K. F. Chung. 2004. Increased expression of transient receptor potential vanilloid-1 in airway nerves of chronic cough. Am.J Respir Crit Care Med. 170:1276-1280.

25. Jeyakumar, A., T. M. Brickman, and M. Haben. 2006. Effectiveness of amitriptyline versus cough suppressants in the treatment of chronic cough resulting from postviral vagal neuropathy. Laryngoscope 116:2108-2112. 
26. Pavord, I. D. and K. F. Chung. 2008. Management of chronic cough. Lancet 371:13751384.

27. Birring, S. S. 2009. Developing antitussives: the ideal clinical trial. Pulm.Pharmacol.Ther. 22:155-158.

28. Morice, A. H. 2010. The cough hypersensitivity syndrome: a novel paradigm for understanding cough. Lung 188 Suppl 1:S87-S90.

29. French, C. T., R. S. Irwin, K. E. Fletcher, and T. M. Adams. 2002. Evaluation of a coughspecific quality-of-life questionnaire. Chest 121:1123-1131.

30. Baiardini, I., F. Braido, O. Fassio, F. Tarantini, M. Pasquali, F. Tarchino, A. Berlendis, and G. W. Canonica. 2005. A new tool to assess and monitor the burden of chronic cough on quality of life: Chronic Cough Impact Questionnaire. Allergy 60:482-488.

31. Birring, S. S. and I. D. Pavord. 2009. Assessment of gender differences in health status with the Leicester Cough Questionnaire (LCQ). Thorax 64:1008-1009.

32. Raj, A. A., D. I. Pavord, and S. S. Birring. 2009. Clinical cough IV:what is the minimal important difference for the Leicester Cough Questionnaire? Handb.Exp.Pharmacol.311320.

33. Fletcher, K. E., C. T. French, R. S. Irwin, K. M. Corapi, and G. R. Norman. 2010. A prospective global measure, the Punum Ladder, provides more valid assessments of quality of life than a retrospective transition measure. J Clin.Epidemiol. 63:1123-1131. 
34. Juniper, E. F., G. H. Guyatt, A. Willan, and L. E. Griffith. 1994. Determining a minimal important change in a disease-specific Quality of Life Questionnaire. J.Clin.Epidemiol. 47:81-87.

35. Morice, A. H., M. S. Menon, S. A. Mulrennan, C. F. Everett, C. Wright, J. Jackson, and R. Thompson. 2007. Opiate therapy in chronic cough. Am.J.Respir.Crit Care Med. 175:312-315.

36. Birring, S. S., T. Fleming, S. Matos, A. A. Raj, D. H. Evans, and I. D. Pavord. 2008. The Leicester Cough Monitor: preliminary validation of an automated cough detection system in chronic cough. Eur.Respir.J. 31:1013-1018.

37. Barry, S. J., A. D. Dane, A. H. Morice, and A. D. Walmsley. 2006. The automatic recognition and counting of cough. Cough. 2:8.

38. Vizel, E., M. Yigla, Y. Goryachev, E. Dekel, V. Felis, H. Levi, I. Kroin, S. Godfrey, and N. Gavriely. 2010. Validation of an ambulatory cough detection and counting application using voluntary cough under different conditions. Cough. 6:3.

39. Birring, S. S., S. Matos, R. B. Patel, B. Prudon, D. H. Evans, and I. D. Pavord. 2006. Cough frequency, cough sensitivity and health status in patients with chronic cough. Respir.Med. 100:1105-1109.

40. Birring, S. S., V. M. Mann, S. Matos, N. Yousaf, C. Wood, T. Fleming, D. H. Evans, A. H. Morice, and I. D. Pavord. 2008. The Leiceseter Cough Monitor. Eur.Respir J 32:530531. 
41. Birring, S. S., S. Matos, R. B. Patel, B. Prudon, D. H. Evans, and I. D. Pavord. 2006. Cough frequency, cough sensitivity and health status in patients with chronic cough. Respir.Med. 100:1105-1109.

42. Yousaf, N., W. Monteiro, D. Parker, S. Matos, S. Birring, and I. D. Pavord. 2010. Longterm low-dose erythromycin in patients with unexplained chronic cough: a double-blind placebo controlled trial. Thorax 65:1107-1110.

43. Dicpinigaitis, P. V., J. B. Dobkin, and J. Reichel. 2002. Antitussive effect of the leukotriene receptor antagonist zafirlukast in subjects with cough-variant asthma. $J$ Asthma 39:291-297.

44. Smith, J., E. Owen, J. Earis, and A. Woodcock. 2006. Effect of codeine on objective measurement of cough in chronic obstructive pulmonary disease. J.Allergy Clin.Immunol. 117:831-835.

45. Woodcock, A., R. L. McLeod, J. Sadeh, and J. A. Smith. 2010. The efficacy of a NOP1 agonist (SCH486757) in subacute cough. Lung 188 Suppl 1:S47-S52. 
Table legends

Table 1. Uncommmon chronic cough causes/associations

Table 2. Cough Hypersensitivity Syndrome: features 


\section{Figure legends}

Figure 1. An approach to the evaluation of airway symptoms: a role for cough reflex Hypersensitivity. 
Table 1. Uncommmon chronic cough causes/associations

Obstructive sleep apnoea

Snoring

Ear wax (Arnold's reflex)

Tonsillar enlargement

Autoimmune disease (hypothyroidism)

Hereditary sensory polyneuropathy

Basidiomycetous fungi

Tracheobronchopathia osteochondroplastica 
Table 2. Cough Hypersensitivity Syndrome: features

Chronic cough duration $>2$ months

Minimal or no sputum production

One of more cough reflex triggers

(cold air, speech, eating, odors such as perfumes)

Urge to cough (tickle or itch) located in throat area

Adverse impact of cough on QOL

Positive cough reflex challenge test (e.g. capsaicin) 
Figure 1. An approach to the evaluation of unexplained airway symptoms: a role for cough reflex hypersensitivity.

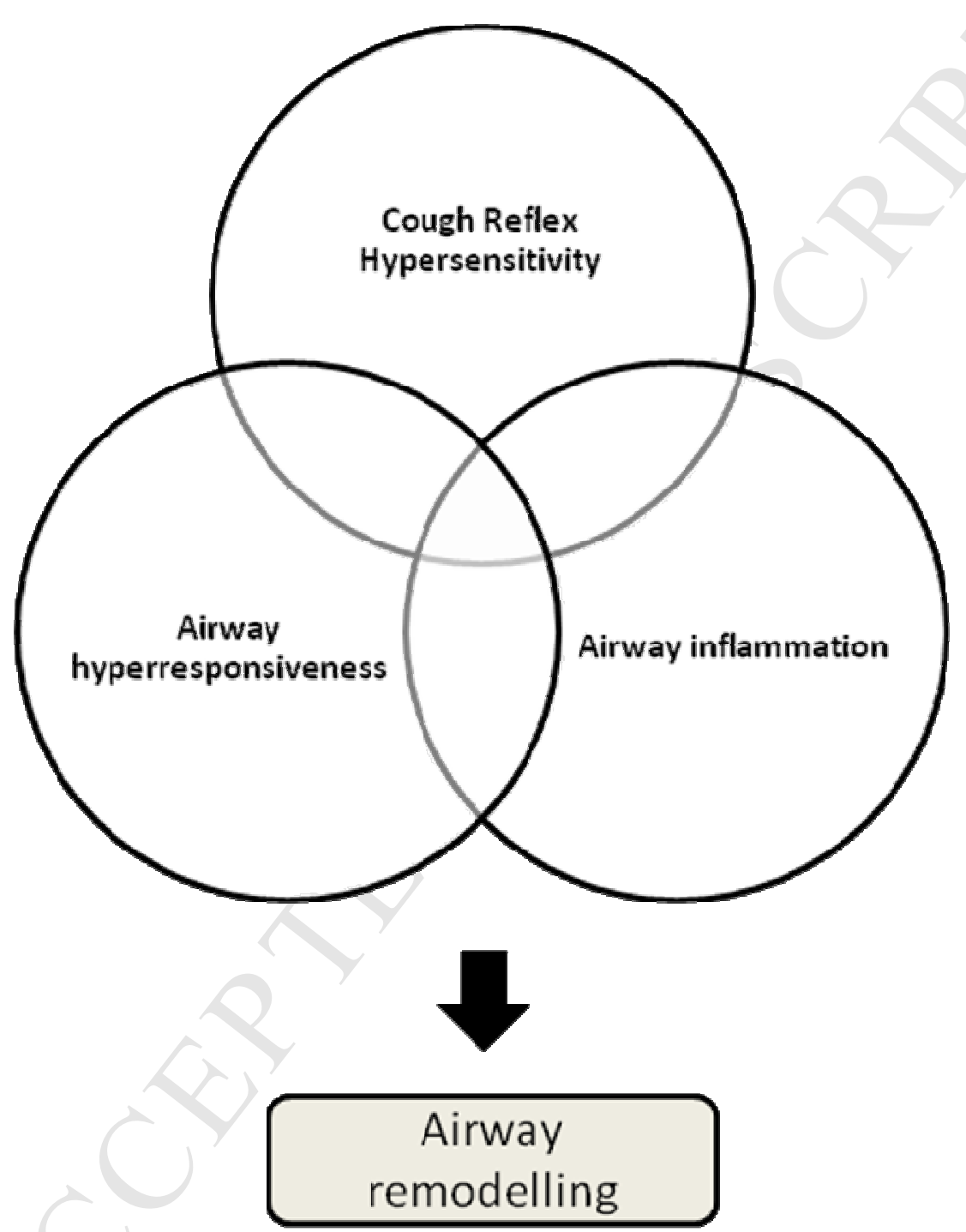

Cough reflex hypersensitivity can co-exist with airway inflammation and hyperresponsiveness in conditions such as asthma and COPD. Cough hypersensitivity syndrome has been proposed as a term for patients with unexplained chronic cough and cough reflex hypersensitivity. 